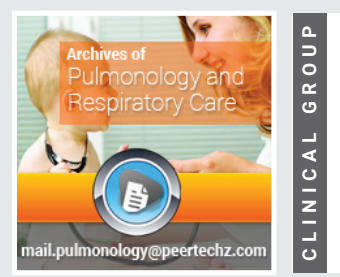

\section{Correlation of clinical,}

\section{radiologic and pathologic}

\section{manifestations with prognosis}

\section{in lung cancer patients}

\section{Halil Yanardag', Cuneyt Tetikkurt ${ }^{2 *}$, Cigdem Papila ${ }^{3}$, Muammer Bilir ${ }^{1}$ and Seza Tetikkurt ${ }^{4}$}

${ }^{1} \mathrm{MD}$, Professor, Department of Internal Medicine Medicine, Cerrahpasa Medical Faculty, Istanbul Cerrahpasa University, Turkey

${ }^{2} \mathrm{MD}$, Professor, Department of Pulmonary Medicine, Cerrahpasa Medical Faculty, Istanbul Cerrahpasa University, Turkey

${ }^{3} \mathrm{MD}$, Professor, Department of Oncology, Cerrahpasa Medical Faculty, Istanbul Cerrahpasa University, Turkey

${ }^{4} \mathrm{MD}$, Professor, Department of Pathology, Demiroglu Bilim University Medical Faculty, Turkey
Received: 13 January, 2019

Accepted: 03 February, 2020

Published: 05 February, 2020

*Corresponding author: Cuneyt Tetikkurt, MD, Professor, Department of Pulmonary Medicine, Cerrahpasa Medical Faculty, Istanbul Cerrahpasa University, Tanzimat Sok. Serkan Apt. No:8/16, 34728, Caddebostan, Istanbul, Turkey, Tel: +90-216-360 19 77; +90-532-381 09 00; Fax: +90-212-587 02 17;

E-mail: tetikkurt@gmail.com

Keywords: Lung cancer; Pulmonary function tests; Prognosis; Pathology

https://www. peertechz.com

Check for updates

\title{
Abstract
}

Many different factors that are extremely variable for each individual affect the survival of lung cancer patients. Prognosis in lung cancer depends upon tumor type, patient immunity and treatment modalities. The objective of our study was to determine the influence of clinical manifestations including the patient symptoms, laboratory findings, imaging modalities and histopathologic tumor features on the prognosis in lung cancer. Another aim was to evaluate whether the collaboration of these data predicted a more accurate prognostic assessment.

A total of 1800 lung cancer patients evaluated at our clinic between 1984 and 2005 years were included in the study. The patients had blood chemistry, chest x-ray, pulmonary function tests, chest computed tomography and histopathology. Prognosis of the patients was evaluated in regard to initial clinical symptoms, performance status, laboratory findings, pulmonary function test results, radiologic, CT and histopathologic manifestations. For patient performance BODE and Karnofsky index were used. Statistical analysis was performed by using Pearson correlation and chi-square tests.

Patient performance status and symptoms had a weak correlation with prognosis. The routine laboratory and the pulmonary function test results were incoherent predictors of survival. Chest x-ray and computed tomography findings displayed a noteworthy correlation for the prognostic outcome. Histopathologic features including tumor type, differentiation profile and microscopic invasive features of the airways, nerves, blood or lymphatic vessels were the hallmark of prognostic evaluation along with immunohistochemistry findings. The above criteria other than the pathologic manifestations showed a poor or a moderate correlation by themselves for an accurate prognostic outcome. Collaboration of the clinical manifestations revealed a significantly high correlation for the prognostic evaluation of lung cancer. The most accurate prognostic determination was achieved in advanced stage cases. The results of our study indicate that the more specific the clinical criteria are considered in a lung cancer patient, the more accurate the prognostic assessment will be since cancer pathogenesis is associated with many different complex mechanisms.

\section{Introduction}

Lung cancer is the most common cancer worldwide accounting for 2.1 million new cases with 1.8 million deaths currently [1]. Approximately 234,030 new cases are estimated to be diagnosed that represent about 13 percent of all cancer diagnoses [2]. In addition, the five-year survival rate of lung cancer is $18.6 \%$ which is lower than the other leading cancer sites, such as colorectal (64.5\%), breast (89.6\%) and prostate (98.2\%) [3]. The five-year survival rate for lung cancer is $56 \%$ in cases with localized disease while only one out of seven lung cancer cases is diagnosed at an early stage. On the other hand, the five-year survival rate is only 5 percent in patients with metastatic lung cancer. Furthermore, almost more than half of the lung cancer patients die within one year after diagnosis [4]. Lung cancer was a rare disease at the start of the twentieth 
century but exposure to carcinogenic agents has caused lung cancer to become a pandemic. It is the most lethal cancer among both men and women in the United States. Lung cancer is also the most frequent cause of cancer death among men, is the second foremost of death among all cancer types and is the second leading cause of cancer death among women worldwide that causes 1.6 million deaths annually which is more than the total number of mortality due to breast, prostate and colon cancer [5-7] . Lung cancer shows significant differences among the individual patients in terms of clinical presentation, histopathology, course, response to treatment and prognosis due to its heterogeneous structure which is the hallmark of its clinical profile. Prognostic outcome and survival of lung cancer patients is mostly relevant to the stage along with the histopathologic features of the tumor. Clinicians should keep in mind that every individual patient with a particular stage of lung cancer does not exhibit an identical treatment response or the same prognosis. There are serious inadequacies in determining the prognostic outcome and directing the treatment modalities concerning the conventional staging system of lung cancer There are many factors like infection, treatment complications and comorbid disease that may adversely effect the prognosis of lung cancer patients other than the tumor itself.

Curative treatment is almost impossible in most of the patients with advanced lung cancer. The overall 5-year survival with lung cancer is only $11.2 \%$ for men and is $13.9 \%$ for women [8-11]. Current studies target to identify patients with earlystage disease to improve life expectancy. Prolonging survival and improving quality of life for patients presenting with advanced lung cancer are also the main concerns of today's research. In these circumstances, studies on the prediction of survival gain great importance that provide guidance in terms of both prognosis and treatment options for the lung cancer.

The aim of our study is to evaluate the effect of the initial symptoms, performance status, laboratory and radiologic manifestations along with the histopathologic tumor features on the prognosis in the lung cancer patients. The second aim of our study was to determine whether the collaboration of these aforementioned criteria would provide a more accurate prognostic determination than by themselves alone.

\section{Materials and methods}

A total of 1800 lung cancer patients as out or inpatients admitted to Cerrahpasa Medical Faculty between 1984 August and 2005 November participated in the study. There were $1640(91.1 \%)$ male and $160(8.8 \%)$ were female patients. Mean age was $59.6 \pm 18.2$ years. Patients with comorbid diseaes and tumors metastatic to the lung were excluded from the study. Primary pleural, thoracic wall soft tissue and bone tumors were also exempted while only patients with primary lung cancer were included. Two hundred forty patients (220/1800; $12.2 \%)$ were non-smokers while 120 (120/1800; 6.6\%) were passive smokers. BODE dyspnea and Karnofsky index were used to determine the performance status of the patients. The initial manifestations including age, gender, smoking habitus, occupational history, symptoms, laboratory findings, radiologic manifestations and histopathologic features were evaluated in regard to the prognostic outcome of the patients by themselves and in collaboration as a new staging system. Immunohistochemical tumor markers including $\mathrm{CF} 5 / 6, \mathrm{CF} / 7$, TTF1, p63, chromogranin and snaptophysin were also analyzed for their contribution to the prognosis of lung cancer.

Patients with metastatic disease were identified by symptoms, laboratory, bone scintigraphy, CT and MR. Specific organ metastasis of lung carcinoma to various organs including brain, liver, bone and adrenal glands was evaluated by neurology or by internal medicine consultation. All the patients were followed-up with three month interval as outpatients. The mean follow-up duration was $128.6 \pm 18.4$ months. For statistical analysis, Pearson correlation test and chi-square were used. Pearson test was carried out to determine the correlation between different variables. Correlation coefficient was nominated as weak $(r<0.3)$, intermediate $(0.3 \leq r \leq 0.7)$ or strong $(r>0.7)$. A $p$ value less than 0.5 revealed statistical significance. The prognostic outcome assessment was appraised due to the survival time as months following initial diagnosis. Prognosis forecast was defined as poor (12-36 months), intermediate (37-60 months) and good (more than 60 months) in regard to disease free time after the initial diagnosis of lung cancer. Statistical analysis and prognostic correlation in regard to gender, age, initial symptoms, smoking habitus, occupational histroy, patient performance index, pulmonary function tests, chest radiologic, thorax computed tomography findings and histopathological features in regard to prognosis was performed. The collaboration of these factors were also analyzed to determine a more precise prognostic outcome.

\section{Results}

Dermographic features of the patients including age, gender, smoking habitus, occupational history along with the laboratory findings and the pulmonary function test results are depicted in Table 1. The initial clinical, laboratory, pulmonary function test and radiologic manifestations along with the histopathologic tumor features with immunohistochemcial staining profile of the patients is shown in Table 2. The most common lung cancer was adenocarcinoma $(36.4 \%, p<0.05)$ followed by squamos cell carcinoma $(23.8 \%, p<0.05)$ cancer in smokers. Adenocarcinoma again had the highest incidence $(25.2 \%, p<0.05)$ while squamos cell carcinoma was the second most frequent $(20.6 \%, p<0.05)$ lung cancer among nonsmokers. There were significant differences in tumor location between smokers and non-smokers. In smokers, the right peripheral and proximal areas were more frequent $(56.4 \%)$ as tumor sites while in non-smokers the tumor was more commonly located in the left peripheral and left proximal areas in $58.2 \%$ of the patients. In female patients adenocarcinoma incidence was significantly $(p<0.05)$ higher than the male patients. There was no statistically significant effect of gender and age relevant to prognosis (Table 3). Weight loss (more than $6 \mathrm{~kg}$ in the last three months), hemoptysis and lassitude were identified as the most common initial manifestations but their prognostic correlation was poor and insignificant. BODE and Karnofsky index were indeterminate indicators for prognosis without any statistical significance. 
Statistical analysis for prognostic correlation status of the patients in regard to gender, age, initial symptoms, smoking habitus, occupational histroy, patient performance index, pulmonary function tests, chest radiologic, thorax computed tomography findings and histopathological features in regard to prognosis is depicted in Table 3. Among the laboratory findings, initial high serum calcium levels and increased serum lactate dehydrogenase levels were the most commonly observed laboratory findings while their correlation with prognosis was insignificant (Table 3). Smoking history in regard to the number of packages-year revealed significant correlation $(r: 0.78, p<0.05)$ with prognosis. Patients with more than 30

Table 1: Demographic and the laboratory findings of the patients.

Dermographics Total \# of patients 1800 Male 1640

\begin{tabular}{|c|c|c|c|}
\hline Age, yr & $64.8 \pm 14.6$ & $68.2 \pm 12.6$ & $58.6 \pm 11.2$ \\
\hline Laboratory & & & \\
\hline ERS $(\mathrm{mm})$ & $32.4 \pm 16.8$ & $36.2 \pm 14.6$ & $28.8 \pm 12.6$ \\
\hline CRP $(\mathrm{mg} / \mathrm{L})$ & $16.2 \pm 6.8$ & $16.4 \pm 8.2$ & $12.4 \pm 8.2$ \\
\hline SGOT (U/L) & $46.8 \pm 26.4$ & $48.2 \pm 16.8$ & $42.6 \pm 18.2$ \\
\hline SGPT (U/L) & $42.6 \pm 18.4$ & $46.8 \pm 14.6$ & $48.6 \pm 14.4$ \\
\hline LDH (U/L) & $298.4 \pm 48.2$ & $288.2 \pm 52.4$ & $278.4 \pm 32.6$ \\
\hline Ca $(\mathrm{mg} / \mathrm{dL})$ & $10.76 \pm 6.4$ & $9.82 \pm 5.72$ & $9.74 \pm 6.58$ \\
\hline Spirometry & & & \\
\hline FEV ${ }_{1} \%$ predicted & $74.6 \pm 18.2$ & $72.8 \pm 14.6$ & $78.6 \pm 14.2$ \\
\hline FVC, \% predicted & $88.2 \pm 14.8$ & $76.4 \pm 16.2$ & $81.2 \pm 13.8$ \\
\hline TLC, \% predicted $_{\text {DLCO/V }}$ \% predicted & $80.6 \pm 14.8$ & $79.4 \pm 14.8$ & $76.4 \pm 14.8$ \\
\hline
\end{tabular}

Data are presented as mean \pm SD or $\%$. FEV : Forced expiratory volume in one second; FVC: Forced vital capacity; TLC: Total lung capacity; DLCO/V: Diffusing capacity divided by the alveolar volume (DLCO/VA).

Table 2: Total number of patients with abnormal clinical manifestations

\begin{tabular}{|c|c|c|c|}
\hline Patient features & Total(\%) & Male(\%) & Female(\%) \\
\hline \# of patients & $1800(100 \%)$ & $1640(91.1 \%)$ & $160(8.9 \%)$ \\
\hline Weight loss & $168(9.7 \%)$ & $130(7.2 \%)$ & $38(23.8 \%)$ \\
\hline Lassitude & $124(6.8 \%)$ & $84(4.6 \%)$ & $40(25 \%)$ \\
\hline BODE index & $62(3.4 \%)$ & $48(2.6 \%)$ & $14(8.8 \%)$ \\
\hline Karnofsky index & $58(3.2 \%)$ & $40(2.2 \%)$ & $18(11.3 \%)$ \\
\hline Biochemistry & $164(9.1 \%)$ & $136(7.6 \%)$ & $28(18 \%)$ \\
\hline Lung function & $324(18 \%)$ & $288(17.5 \%)$ & $36(22.5 \%)$ \\
\hline Chest x-ray findings & $844(52.8 \%)$ & $782(43.4 \%)$ & $62(38.8 \%)$ \\
\hline Lung CT findings & $1218(67.6 \%)$ & $1120(70 \%)$ & $98(61.2 \%)$ \\
\hline Pathologic findings & $1362(75.6 \%)$ & $1150(50.9 \%)$ & $112(70 \%)$ \\
\hline Immunohistochemisty & $1180(65.6 \%)$ & $1060(66.2 \%)$ & $120(75 \%)$ \\
\hline *SBPPFXCPI & $1425(79.2 \%)$ & $1300(81.2 \%)$ & $125(78.1 \%)$ \\
\hline
\end{tabular}

*SBPFXCPI: collaboration of clinical and laboratory findings.

S: Symptoms; B: Biochemistry; P: Performance status [BODE and Karnofsky]; F Pulmonary function tests; X: Chest x-ray; C: Computed tomography; P: Pathology (tumor type and differentiation; Blood vessel, lymphatic and nerve invasion), I: Immunochemistry findings (Chromogranin CF5/6, CF/7, TTF1, p63 and snaptophysin) packages-year history revealed a noteworthy higher incidence of small cell carcinoma $(p<0.05)$ and undifferentiated tumor cell profile $(p<0.05)$. The high burden of smoking showed an explicit correlation with poor prognosis $(r: 082, p<0.01)$.

Chest $\mathrm{x}$-ray and computed tomography of the thorax findings demonstrated significant finding relevant to prognosis in lung cancer patients. A large tumor size (diameter $>3.0 \mathrm{~cm}$ ), irregular or spicular tumor borders and a cavity wall thickness more than $15 \mathrm{~mm}$ were significant manifestations for outcome revealing a high correlation (Table 3) with prognosis. Lower lobe tumors had the worst prognosis regardless of the histolopathogic type ( $r: 0.74, p<0.05$ ) . Considering the histolopathologic type, small cell followed by large cell had the worst prognosis prognostic outcome ( $r: 0.92 ; p<0.01$ and $r: 0.84 ; p<0.01)$ among our patients. The prognostic outcome exhibited an increasing poor trend along with higher stages and advanced histopathologic tumor features. Tumor differentiation and vascular, lymphatic and nerve invasion provided the most accurate prognostic (Table 3) determination. The most definitive prognostic assessment in lung cancer patients was obtained when all the aforementioned criteria including the initial symptoms, the laboratory, the radiologic and the histopathologic findings were evaluated in collaboration.

\section{Discussion}

Many different and variable factors can affect the survival and prognostic outcome of lung cancer patients. Genetics, immunity, comorbid diseases, tumor stage, tumor type and differentiation determine the prognosis in lung cancer. Since many individual and pathogenetic factors play a role in the

Table 3: Statistical analysis and correlation of clinical manifestations with lung cancer prognosis.

\begin{tabular}{|c|c|c|c|c|}
\hline Patient features and laboratory findings & BP & IP & GP & p value \\
\hline Age (years) & $r: 0.12$ & $r: 0.11$ & $r: 0.14$ & $<0.32$ \\
\hline Gender & $r: 0.16$ & $r: 0.14$ & $r: 0.18$ & $<0.26$ \\
\hline 0-20 packages-year & $r: 0.10$ & $r: 0.15$ & $r: 0.68$ & $<0.05$ \\
21-40 packages-year & $r: 0.38$ & $r: 0.12$ & $r: 0.54$ & $<0.05$ \\
41-60 packages-year & $r: 0.72$ & $r: 0.28$ & $r: 032$ & $<0.05$ \\
\hline >60 packages-year & $r: 0.78$ & $r: 0.68$ & $r: 0.62$ & $<0.05$ \\
\hline Initial symptom analysis & $r: 0.10$ & $r: 0.16$ & $r: 0.12$ & $<0.24$ \\
\hline Patient performance index & $r: 0.14$ & $r: 0.12$ & $r: 0.16$ & $<0.30$ \\
\hline Serum biochemistry & $r: 0.12$ & $r: 0.16$ & $r: 0.18$ & $<0.36$ \\
\hline PFT & $r: 0.11$ & $r: 0.10$ & $r: 0.14$ & $<0.28$ \\
\hline Chest x-ray & $r: 0.56$ & $r: 0.68$ & $r: 0.64$ & $<0.05$ \\
\hline Computed tomography manifestations & $r: 0.78$ & $r: 0.74$ & $r: 0.82$ & $<0.05$ \\
\hline Pathologic features & $r: 0.82$ & $r: 0.86$ & $r: 0.84$ & $<0.01$ \\
\hline Immunohistochemistry & $r: 0.80$ & $r: 0.84$ & $r: 0.88$ & $<0.01$ \\
\hline *SBPFXCPI & $r: 0.92$ & $r: 0.90$ & $r: 0.94$ & $<0.01$
\end{tabular}

PFT: Pulmonary Function Test.

*SBPFXCPI: Collaboration of clinical and laboratory findings (S: Symptoms; B: Biochemistry; P: Performance status [BODE and Karnofsky]; PF: Pulmonary function tests; X: Chest X-ray; C: Computed tomography; P: Pathology; I: Immunochemistry findings).

$\chi^{2}$ test, ANOVA, Kruskal-Wallis test and Bonferronni corrected two way Mann-Whitney test were used for statistical analysis. Pearson test was used for correlation analysis between prognosis and different clinical variables. 
pathogenesis of lung cancer, the more the specific patient criteria is used to determine survival, the more accurate the prognostic evaluation will be. Survival determination will reveal less explicit results if prognostic evaluation is done by depending upon merely few factors. The results of our study indicate that the most definitive and accurate prognostic outcome determination can only be achieved by the collaboration of all the relevant clinical factors. These factors include the laboratory results, patient symptoms, radiologic findings and pathologic manifestations consisting of tumor type, differentiation and histopathologic features. As the results of our study indicated most of these criteria were insignificant determinants of prognosis by themselves and revealed a weak or a moderate correlation on their own except the amount of cigarettes smoked and the histopathologic tumor features. On the other hand, liaison of all these entities in unison revealed an indisputably accurate and definitive assessment for the prognostic outcome of lung cancer patients.

There was a significant correlation between poor prognosis and cigarette burden. The incidence of small cell and undifferentiated tumor profiles were significantly higher in patients who smoked over 30 pack years. Poor prognosis and higher mortality rates can probably be explained by the intense carcinogen exposure due to excessive smoking. On the other hand, it is a fact that other comorbid effects of smoking such as atherosclerosis, hypertension, gastric, bladder and colon carcinoma may also have an impact on the poor prognostic outcome and higher mortality rates. Radiologically, higher tumor size and presence of spicular extensions on chest $\mathrm{x}$-ray and CT exhibited a significant negative prognostic factor with a moderate correlation with prognosis. Histopathologic vascular, lymphatic or nerve invasion were an extremely crucial and definitive histopathologic features for poor prognosis. The tumor type and differentiation were also crucial predictors of survival and revealed significant correlation with prognosis as undifferentiated tumors and small cell carcinoma followed by adenocarcinoma showed the worse prognosis. On the other hand, the most evident hallmark of tumor prognosis was achieved when all the above criteria were collaborated. Because pathogenesis of lung cancer is associated with many variable and complex factors, an accurate determination of prognosis will be only carried out if numerous and patient specific criteria are taken into account to determine the outcome of lung cancer patients as the results of our study explicitly indicated.

The more pronounced the undifferentiated status of the tumor, the greater will be the tumor aggressiveness leading to poor survival. The tumor type is another significant predictor of survival that was the worst prognostic factor for the small cell carcinoma type. CT findings of higher dimension including axial and sagittal diameters along with the spicular extensions were the hallmark of advanced tumor that were associated with a poor patient outcome. Pathological evidence of vascular, lymphatic or nerve invasion by tumor cells was another manifestation for decreased survival revealing an advanced disease status that indicated the microscopic tumor cell invasion and metastastaic potential. The collaboration of tumor associated clinical factors we introduced determined the prognostic outcome of lung cancer patients more accurately than any individual criteria by itself. As these factors are in a way indirect indicators of advanced or aggressive disease that reveal the different aspects of the tumor status along with metastastic potency which is the hallmark of survival. Consequently, the collaboration of patient specific factors provided a much more accurate and significant prognostic data than the contribution of each factor alone.

The laboratory results revealed a weak correlation with the prognosis of the lung cancer patients due to their low sensitivity and specificity that may also be associated with many other disorders. Because the tumor pathogenesis and development is very complex, it is conspicuous that as more specific factors are incorporated for prognostic evaluation, the more accurate the prognostic assessment will be.

We are aware of the fact that multiple factors play a prognostic role in the survival of lung cancer patients. The small patient population may be considered as the weak side of our study. Our study consisted of only Caucassian people. Therefore, it is a necessity to perform multiple studies with large population sizes including as many as different patient types with discriminating genetic and hereditary features The inclusion of PET/CT manifestations in the study is a factor that may contribute significantly to the accuracy of the prognostic evaluation but PET/CT was not available at time of this research. Lack of molecular studies concerning tumor structure is another vulnerable side of this study. If EGFR, ALK and ROS molecular biomarkers were included in the study, the prognostic evaluation of lung cancer patients could have reached a more precise and definitive extent.

The well known fact that the pathogenesis of lung cancer is sophisticated and obscure depending upon many factors including tumor type, immunity and genetics of the patient along with social and environmental features justifies the results of our current study. Due to this multifaceted tumor structure, we believe that prognostic studies would establish more accurate results if and only if more relevant clinical or tumor specific coefficients are included in the assessment of prognostic outcome. The molecular and the genetic pathways of lung cancer are the distinctive features of lung cancer cell proliferation. In current practice, only a few of the numerous identified molecular targets are used in clinical practice other than the clinical trials. Molecular biology of lung cancer appears to be the hallmark and the most significant marker of patient prognosis. For lung cancer treatment, blocking tumor growth by targeting the surrounding angiogenesis, protumorigenic growth factor activation, anti-apoptotic cascades and other cancer-promoting signal transduction events are extremely important and incredibly effective for prolonging patient survival [12-16]. The heterogeneous complex nature of lung cancer makes it difficult for us to interpret and predict the prognostic outcome accurately. To provide new insights to tumor prognosis data obtained in very diverse patient populations are required. As histopathology and molecular pathways of lung cancer are delineated, we would be able to stratify patients based on a refined understanding of their histology that may more precisely ascertain patient survival. 
modalities were unavailable at the time of our study. Another missing aspect of our study may be considered as the exclusion of individuals with comorbid diseases. Since comorbid diseases effect patient survival independant of lung cancer, their exclusion would only reveal the absolute effect of lung cancer on survival thereby providing a more accurate determination of prognosis.

The targeted treatment in lung cancer aims for specific genes, proteins or the tissue environment that contributes to cancer growth and survival. The treatment includes inhibiting angiogenesis, epidermal growth factor receptor and other genes like ALK, ROS1 and NRTK while immunotherapy restores the immune function of the patient. As these treatment modalities indicate lung cancer is a complex disease consisting of multiple pathogenetic mechanisms. It is well known that some of the patients respond to these treatment options while in others there is no response. A similar treatment response is encountered in the treatment of lung cancer with conventional drug treatment. This clinical profile clearly demonstrates how complex the pathogenesis of lung cancer is. Our study results suggest that to determine an accurate prognostic assessment in lung cancer patients all available clinical factors should be considered and appraised. It is explicit that as the number of tests used for diagnosis increases, the diagnostic specificity and sensitivity will reach to more unequivocal consequences.

It is well known that there are some significant limitations of the new TNM staging system [17-20]. The data lacks details in regard to details of the tumor structure and radiologic manifestations including lymphangitis carcinomatosis. The Standart Uptake Value (SUV) has not been incorporated as well as the absence of data for immunohistochemistry and molecular genetics $[21,22]$. Tumor size and the other vital CT manifestations of lung cancer are not included in the current TNM system that appears to be a fundamental deficiency. The default TNM system lacks specific evaluation of the histopathologic tumor features like lymphatic and blood vessel infiltration of tumor cells which appears to be one of the most crucial and noteworthy determinant for the survival of lung cancer patients. Consequently, TNM system has limited utility for estimating the prognostic outcome of lung cance $[20,23,24]$. The main reason for this insufficient determinant of prognostic assessment is the lack of specific patient data in individual bases that should have included every detail of the patient profile. Our prognostic assessment protocol includes the most crucial factors for an unequivocal assessment of the prognostic outcome of the lung cancer patients. Another important aspect of this study is, the more individualization of personal profile and the more specific data entry for any patient, the more accurate and the more definitive the prognostic assessment will be.

\section{Conclusion}

Lung cancer has many sophisticated aspects comprising distinctive pathogenetic and pathogologic mechanisms with disease course or survival. Prognostic outcome depends upon numerous factors involving tumor type and differentiation, treatment modalities, immune and genetic status of the individual patient. Our study has explicitly established that an accurate survival assessment necessitates an absolute individual patient analysis including the greatest number of prevalant factors of the patient profile comprising all the clinical, laboratory, pathology and imaging data. The prognostic evaluation of any lung cancer patient should be tailored according to the specific individual patient cognisance. The more unique and the more the specific clinical factors are appraised individually for a patient, the more accurate and the more definitive the prognostic assessment of lung cancer prognosis will be. Smoking burden appeared as one of the most significant factors in the prognostic outcome of patients. A third noteworthy outcome for this research is relevant to the advanced lung cancer patients with a poor prognostic outcome in which the new assessment system appears to be the most successful. Consequently, this new protocol has introduced a significantly accurate and undeniable data for the unequivocal evaluation of the prognostic outcome for the lung cancer patients.

\section{Author contributions}

Halil Yanardag has performed patient data collection and evaluation.

Cuneyt Tetikkurt has designed the study and wrote the manuscript.

Cigdem Papila has contributed to organization of patient files and preparation of references.

Muammer Bilir has performed the statistical analysis.

Seza Tetikkurt reviewed the pathologic aspects of lung cancer and histopathologic results for the manuscript.

\section{References}

1. Centers For Disease Control And Prevention (2017) National Center For Health Statistics. CDC WONDER On-Line Database, Compiled from Compressed Mortality File 1999-2016 Series 20 No. 2

2. Siegel RL, Miller KD, Jemal A (2018) Cancer Statistics, 2018. CA: A Cancer Journal for Clinicians 68: 7-30. Link: http://bit.ly/2GXKVLa

3. U.S. National Institute Of Health, National Cancer Institute. SEER Cancer Statistics Review, 1975-2015.

4. Torre LA, Siegel RL, Jemal A (2016) Lung Cancer Statistics. Adv Exp Med Biol 893: 1-19. Link: http://bit.ly/3bf4LiT

5. Mao Y, Yang D, He J, Krasna MJ (2016) Epidemiology of Lung Cancer. Surg Oncol Clin N Am 25: 439-445. Link: http://bit.ly/20tcArs

6. Nasim F, Sabath BF, Eapen GA (2019) Lung Cancer. Med Clin North Am 103 463-473. Link: http://bit.ly/2SkTXqR

7. Dela Cruz CS, Tanoue LT, Matthay RA (2011) Lung cancer: epidemiology, etiology, and prevention. Clin Chest Med 32: 605-644. Link: http://bit.ly/2OvaSGI

8. Molina JR, Yang P, Cassivi SD (2008) Non-small cell lung cancer: epidemiology, risk factors, treatment, and survivorship. Mayo Clin Proc 83: 584-594. Link: http://bit.ly/31rQpqX

9. Gatta G, Mallone S, van der Zwan JM (2013) Cancer prevalence estimates in Europe at the beginning of 2000. Ann Oncol 24: 1660-1666. Link: http://bit.ly/2SiqiyO 
10. Alberg AJ, Brock MV, Samet JM (2005) Epidemiology of lung cancer: looking to the future. J Clin Oncol 23: 3175-3185. Link: http://bit.ly/2twtkHI

11. Alberg AJ, Ford FG, Samet JM (2007) Epidemiology of lung cancer: ACCP evidence-based clinical practice guidelines ( $2^{\text {nd }}$ edition). Chest 132: 29S-55S Link: http://bit.ly/2v4fYCA

12. Hirsch FR, Scagliotti GV, Mulshine JL, Kwon R4, Curran WJ, et al. (2017) Lung cancer: current therapies and new targeted treatments. Lancet 389: 299-311. Link: http://bit.ly/36W2shr

13. Saito M, Shiraishi K, Goto A, Suzuki H, Kohno T, et al. (2018) Development of targeted therapy and immunotherapy for treatment of small cell lung cancer. Jpn J Clin Oncol 48: 603-608. Link: http://bit.ly/37VdKUx

14. Pan Z, Wang K, Chen Q, Zheng X, Song Z, et al. (2020) SFI Enhances Therapeutic Efficiency of Gefitinib: An Insight into Reversal of Resistance to Targeted Therapy in Non-small Cell Lung Cancer Cells. J Cancer 11: 334-344. Link: http://bit.ly/36W2VjH

15. Pruis MA, Geurts-Giele WRR, von der TJH, Meijssen IC, Dinjens WNM, et al (2019) Highly accurate DNA-based detection and treatment results of MET exon 14 skipping mutations in lung cancer. Lung Cancer 18: 46-54.

16. Gibert J, Clavé S, Hardy-Werbin M, Taus Á, Rocha P, et al. (2019) Concomitant genomic alterations in KRAS mutant advanced lung adenocarcinoma. Lung Cancer 5: 42-45.

17. Osarogiagbon RU, Smeltzer MP, Faris N, Rami-Porta R, Goldstraw P, et al (2016) Comment on the Proposals for the Revision of the N Descriptors in the Forthcoming Eighth Edition of the TNM Classification for Lung Cancer. J Thorac Oncol 11: 1612-1614. Link: http://bit.ly/2ulZo0L
18. Rami-Porta R, Asamura H, Travis WD, Rusch VW (2017) Lung cancer-major changes in the American Joint Committee on Cancer eight edition staging manual. CA Cancer J Clin 67: 138-155. Link: http://bit.ly/2RXtSzo

19. Goldstraw P, Crowley J, Giroux DJ, Groome PA, et al. (2006) IASLC International Staging Project. The IASLC International Staging Project on Lung Cancer. J Thorac Oncol 1: 281-286.

20. Rami-Porta R, Goldstraw P (2010) Strength and weakness of the new TNM classification for lung cancer. Eur Respir J 36: 237-239. Link: http://bit.ly/37XYAOU

21. Detterbeck FC (2018) The eight edition TNM stage clasification for lung cancer: What does it mean on the main street ? J Thorac Cardiovasc Surg 155 356-359. Link: http://bit.ly/31oZ6CA

22. Detterbeck FC (2019) Are We There Yet?: Understanding Differences in Rates of Resection of Clinical Stage I Lung Cancer. Chest 155: 7-8. Link: http://bit.ly/36Y5lyj

23. Nicholson AG, Tsao MS, Travis WD, Patil DT, Galateau-Salle F, et al. (2018) Eighth Edition Staging of Thoracic Malignancies: Implications for the Reporting Pathologist. Arch Pathol Lab Med 142: 645-661. Link: http://bit.ly/2uj56R2

24. Detterbeck FC, Chansky K, Groome P, Bolejack V, Crowley J, et al. (2016) IASLC Staging and Prognostic Factors Committee, Advisory Boards, and Participating Institutions. The IASLC Lung Cancer Staging Project: Methodology and Validation Used in the Development of Proposals for Revision of the Stage Classification of NSCLC in the Forthcoming (Eighth) Edition of the TNM Classification of Lung Cancer. J Thorac Oncol 11: 1433-1446. Link: http://bit.ly/2RX6qCj

\section{Discover a bigger Impact and Visibility of your article publication with} Peertechz Publications
Highlights

* Signatory publisher of ORCID

* Signatory Publisher of DORA (San Francisco Declaration on Research Assessment)

* Articles archived in worlds' renowned service providers such as Portico, CNKI, AGRIS, TDNet, Base (Bielefeld University Library), CrossRef, Scilit, J-Gate etc.

* Journals indexed in ICMJE, SHERPA/ROMEO, Google Scholar etc.

* OAI-PMH (Open Archives Initiative Protocol for Metadata Harvesting)

* Dedicated Editorial Board for every journal

* Accurate and rapid peer-review process

* Increased citations of published articles through promotions

* Reduced timeline for article publication

Submit your articles and experience a new surge in publication services (https://www.peertechz.com/submission).

Peertechz journals wishes everlasting success in your every endeavours.

Copyright: (c) 2020 Yanardag H, et al. This is an open-access article distributed under the terms of the Creative Commons Attribution License, which permits unrestricted use, distribution, and reproduction in any medium, provided the original author and source are credited. 